\title{
Is Robot-Assisted Surgery Really Scarless Surgery? Immediate Reconstruction with a Jejunal Free Flap for Esophageal Rupture after Robot-Assisted Thyroidectomy
}

\author{
Seong Hoon Park ${ }^{1}$, Joo Hyun Kim ${ }^{1}$, Jun Won Lee ${ }^{1}$, Hii Sun Jeong ${ }^{1}$, Dong Jin Lee ${ }^{2}$, \\ Byung Chun Kim ${ }^{3}$, In Suck Suh ${ }^{1}$ \\ ${ }^{1}$ Department of Plastic and Reconstructive Surgery, Kangnam Sacred Heart Hospital, Seoul; ${ }^{2}$ Department of Otorhinolaryngology-Head and \\ Neck Surgery, Hallym University Medical Center, Seoul; ${ }^{3}$ Department of Surgery, Kangnam Sacred Heart Hospital, Hallym University \\ College of Medicine, Seoul, Korea
}

Esophageal perforation is a rare but potentially fatal complication of robot-assisted thyroidectomy (RAT). Herein, we report the long-term outcome of an esophageal reconstruction with a jejunal free flap for esophageal rupture after RAT. A 33-year-old woman developed subcutaneous emphysema and hoarseness on postoperative day 1 following RAT. Esophageal rupture was diagnosed by computed tomography and endoscopy, and immediate surgical exploration confirmed esophageal rupture, as well as recurrent laryngeal nerve injury. We performed a jejunal free flap repair of the 8-cm defect in the esophagus. End-to-side microvascular anastomoses were created between the right external carotid artery and the jejunal branches of the superior mesenteric artery, and end-to-end anastomosis was performed between the external jugular vein and the jejunal vein. The right recurrent laryngeal nerve injury was repaired with a 4-cm nerve graft from the right ansa cervicalis. Esophagography at 1 year after surgery confirmed that there were no leaks or structures, endoscopy at 1 year confirmed the resolution of vocal cord paralysis, and there were no residual problems with swallowing or speech at a 5-year follow-up examination. RAT requires experienced surgeons with a thorough knowledge of anatomy, as well as adequate resources to quickly and competently address potentially severe complications such as esophageal rupture.

Keywords Jejunum / Microsurgery / Free tissue flaps
Correspondence: In Suck Suh Department of Plastic and Reconstructive Surgery, Kangnam Sacred Heart Hospital, Hallym University College of Medicine, 1 Singill-ro, Yongdeongpo-gu, Seoul 07441 , Korea

Tel: $+82-2-829-5182$

Fax: +82-2-847-5183

E-mail: sismdps@hallym.or.kr

Received: 1 Jun 2017 • Revised: 18 Jul 2017 • Accepted: 30 Jul 2017

pISSN: 2234-6163・elSSN: 2234-6171・https://doi.org/10.5999/aps.2017.00941・Arch Plast Surg 2017;44:550-553

This article was presented as a poster at the Korean Society for Microsurgery on November 5, 2016 in Seoul, Korea.

\section{INTRODUCTION}

The robotic surgical approach for minimally invasive thyroid surgery has been well described by Korean surgeons and has en- tered into widespread use in Asia [1]. There are several advantages of performing a surgical procedure using a robotic system, such as increased precision and fine motor scaling without tremors, magnification of the operative field, 3-dimensional vi- 
sion, and better cosmetic satisfaction with minimal scarring [1] Although many studies have reported favorable surgical outcomes of robot-assisted thyroidectomy (RAT), it is very important that RAT is performed by experienced surgeons with appropriate skills, accurate technique with surgical instruments, and a thorough knowledge of anatomy, as well as adequate resources to quickly and competently address potentially urgent situations and severe complications. Moreover, RAT showed a significantly longer operating time with a higher cost than open surgery, and many complications have been observed after RAT, such as symptomatic hypocalcemia, recurrent laryngeal nerve injury, hematoma formation, chyle leakage, Horner syndrome, carotid artery injury, and trachea injury. Perforation of the cervical esophagus is a rare but recognized complication of RAT. The clinical features of this injury are variable, and may include apparent signs of esophageal leakage, such as pain, swelling, and tenderness of the neck; cough; dysphagia; aspiration; fever of unknown origin; leukocytosis; and unexplained tachycardia [2].

The primary goals of reconstruction are wound closure and the protection of vital structures, along with the restoration of anatomy, normal breathing, normal dynamic swallowing without aspiration, and speech within an acceptable healing time $[2,3]$. Although a wide variety of reconstructive methods have been developed, the most commonly reported method is the jejunal free flap [3]. First described in 1959 by Seidenberg, the free jejunal flap was introduced as a reliable and reproducible method of re-establishing the mucosal conduit, thereby preserving the patient's ability to swallow [3]. The jejunal flap is a useful substitute for the esophagus because it resembles the anatomy of the esophagus, maintains peristalsis, and is usually free of intrinsic disease [4]. Thus, the jejunal flap has become a popular and effective technique [5].

Herein, we report the long-term outcome of an esophageal reconstruction with a jejunal free flap that was performed for esophageal rupture after RAT.

\section{Fig. 1. Emphysema and perforation on CT and EGD}

The patient developed subcutaneous emphysema in the neck after robot-assisted thyroidectomy. Esophageal perforation was seen on computed tomography (CT) and esophagogastroduodenoscopy (EGD).

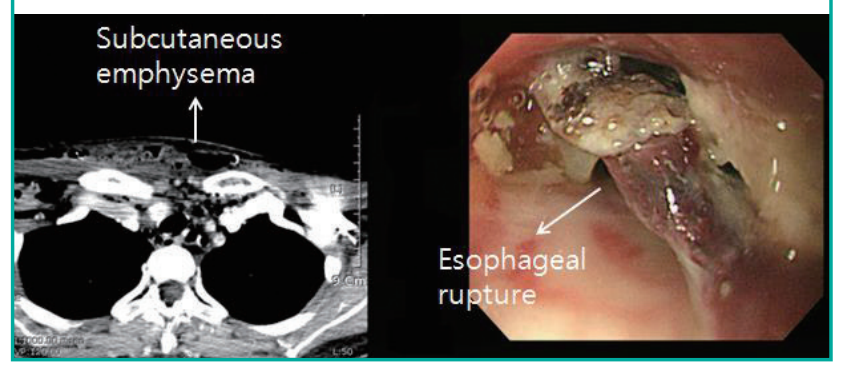

\section{CASE}

A 33-year-old woman who was diagnosed with thyroid cancer of the right lower lobe measuring $6 \mathrm{~mm}$ (T1M0N0, stage I) developed subcutaneous emphysema and hoarseness on postoperative day 1 after RAT. Esophageal rupture was diagnosed by computed tomography and endoscopy (Fig. 1). Surgical exploration confirmed esophageal rupture and recurrent laryngeal nerve injury, and a jejunal free flap repair of the esophagus was performed. The loop of jejunum to be used was determined according to the size of the esophageal defect, which measured 8 $\mathrm{cm}$. After standard preoperative procedures, the jejunum was exposed through an upper midline abdominal incision. The length of bowel used was a minimum of 30 to $35 \mathrm{~cm}$ from the ligament of Treitz, and the mesentery and principal vessels were meticulously dissected [5]. The bowel was divided by a stapler at both ends and the perfusion of the jejunum was checked before ligation of the pedicle [5]. The harvested flap was transferred to the neck area and subsequent microsurgical anastomoses were done by the plastic surgery team. Specifically, end-toside microvascular anastomoses were performed between the right external carotid artery and the jejunal branches of the superior mesenteric artery, and an end-to-end anastomosis was created between the external jugular vein and the jejunal vein (Figs. 2, 3). The right recurrent laryngeal nerve was then repaired with a $4-\mathrm{cm}$ nerve graft from the right ansa cervicalis. Abdominal closure and small bowel anastomosis were performed by general surgeons. At a 5-year postoperative follow-up examination, the patient had no residual functional problems with swallowing or speech, and there was no evidence of esoph-

Fig. 2. Harvest of the jejunum and vessels

A portion of the second arcade of the jejunum measuring approximately $15 \mathrm{~cm}$ was harvested along the jejunal branch of the superior mesenteric artery and vein.

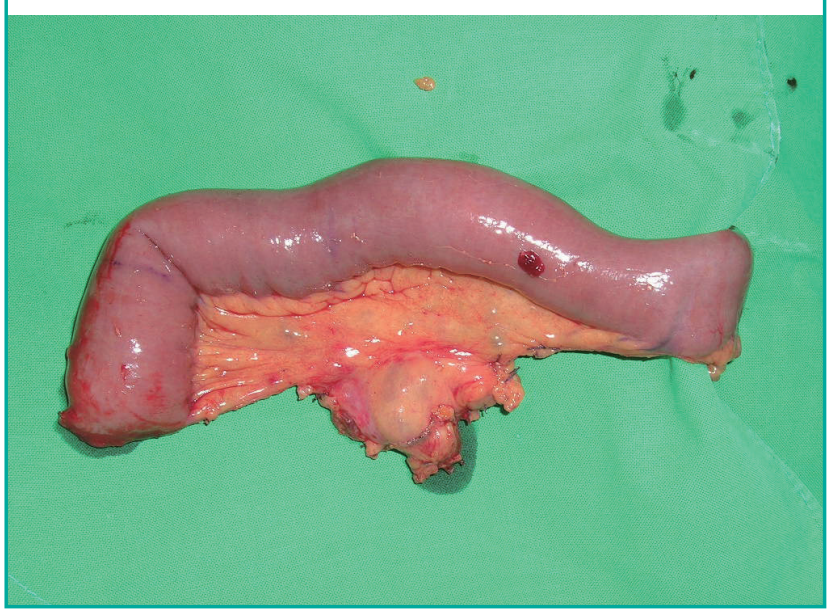




\section{Fig. 3. Jejunal flap and microvascular anastomosis}

During exploration, esophageal rupture and recurrent laryngeal nerve injury was discovered. We performed a jejunal free flap for the esophageal defect and microvascular anastomosis was performed with the external carotid artery, and external jugular vein.
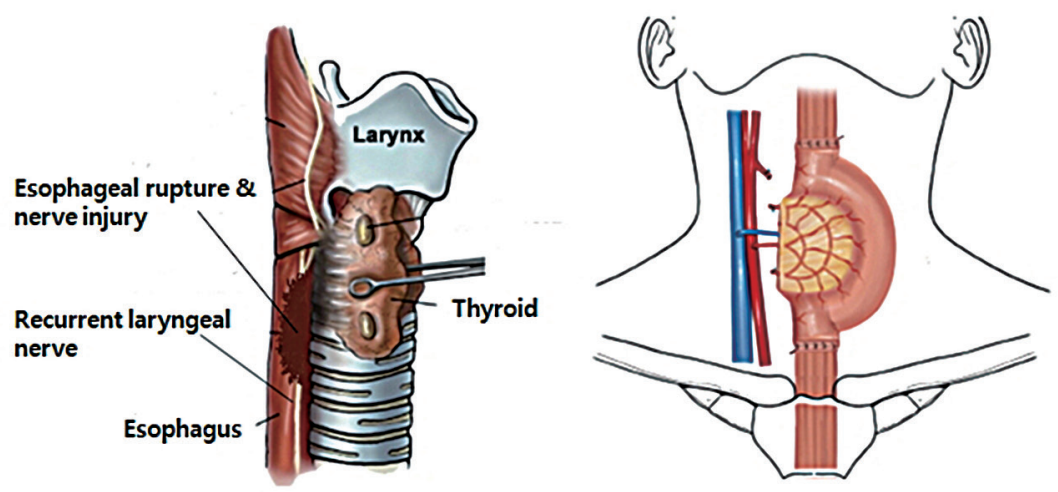

ageal leakage or stricture on esophagography. The patient had recovered her voice without hoarseness by 5 months postoperatively, and endoscopy at 1 year postoperatively showed improvement of the vocal cord paralysis (Fig. 4). The patient underwent a scar revision, and a fine linear scar remained visible on the anterior aspect of the neck at year 5 .

\section{DISCUSSION}

It is essential that RAT is performed by experienced surgeons with a thorough knowledge of anatomy. If complications such as esophageal rupture occur, resources and surgical teams should be available for immediate management. In our patient, immediate reconstruction with a jejunal free flap was possible and the patient had a good outcome. Occult esophageal perforation after surgery or penetrating or blunt trauma may head to severe mediastinitis, sepsis, and death [6]. Traumatic perforations of the cervical esophagus account for $20 \%$ to $40 \%$ of all esophageal injuries [7]. A mortality rate of between $4 \%$ and $50 \%$ has been reported [2], and early diagnosis and treatment are known to be associated with improved survival [8].

Perforation of the cervical esophagus should be suspected in patients with neck or throat pain, dysphagia, odynophagia, unexplained aspiration or choking, fever, localized neck tenderness, induration, hemoptysis, or the presence of subcutaneous emphysema [9]. Plain radiographs of the cervical spine and a chest X-ray may show subcutaneous emphysema and pneumomediastinum. Definitive diagnosis and localization of the perforation is usually established by a contrast esophagogram [9], but this was not indicated in our case because the postoperative leakage was obvious.

Esophageal perforations can often be repaired by double-layer imbricating sutures $[2,10]$. However, this technique results in esophageal strictures in up to $54 \%$ of patients [10], while nonsurgical treatment of esophageal perforation has been related to

\section{Fig. 4. Results: a linear scar with no complications}

(A) Esophagogastroduodenoscopy shows flap continuity without necrosis. Esophagography shows no evidence of leakage or stricture. Endoscopy shows resolution of vocal cord paralysis, and the patient's hoarseness had improved. (B) Scar revision resulted in a fine linear scar on the anterior aspect of the neck.

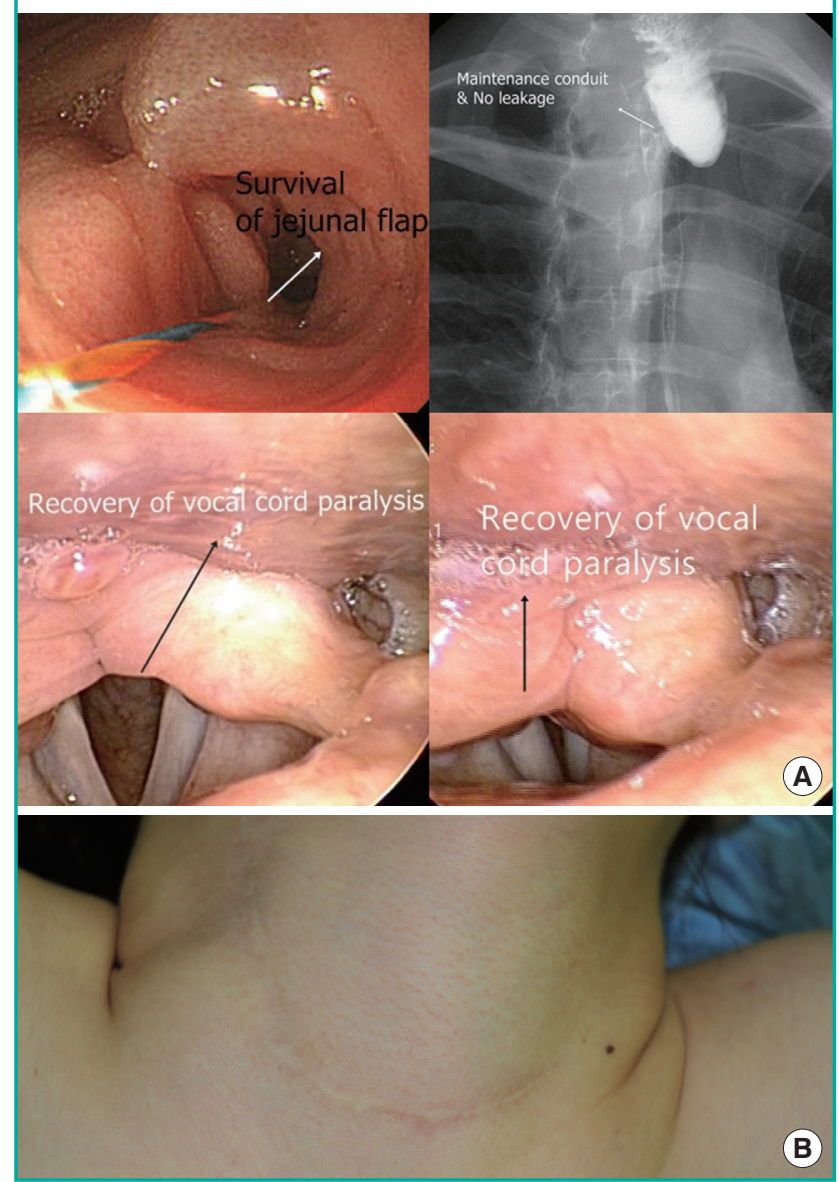

a high incidence of fistula formation requiring prolonged tube feeding [6].

There are various methods for reconstruction of the cervical esophagus. The jejunal free flap has been used successfully for 
reconstructions of the pharynx and esophagus in patients with tumors, and the tubed free radial forearm flap is also used frequently for these patients $[11,12]$. Other methods, such as the submental island flap and the pectoralis major myocutaneous flap, might also be suitable for cervical esophageal reconstruction [2].

The free jejunal flap has quickly evolved to become the method of choice for reestablishing the mucosal conduit of the esophagus and pharynx [13]. Success rates ranging from $95 \%$ to $100 \%$ have been reported for this technique, and major donorsite related complications such as abdominal wound dehiscence, bowel obstruction, or bleeding from the mesenteric root are rare $[14,15]$. Jejunal flaps have many advantages, such as radiation tolerance, restoration of functional swallowing, and voice restoration [13]. As noted in this case, the patient had no residual functional deficiencies in either speech or swallowing at a 5-year follow-up visit.

In summary, esophageal perforation is a rare but recognized complication after robot-assisted thyroid surgery, and can often be managed by primary suture repair. However, this technique is associated with a high incidence of secondary strictures, and local flaps may be more useful for repairing larger defects. The free jejunal flap has consistently proven to be a reliable method that is associated with low complication rates and full restoration of function. Ongoing improvements in technique and new developments should bring further improvements.

\section{CONFLICT OF INTEREST}

No potential conflict of interest relevant to this article was reported.

\section{PATIENT CONSENT}

The patient provided written informed consent for the publication and the use of their images.

\section{REFERENCES}

1. Piccoli M, Mullineris B, Santi D, et al. Advances in robotic transaxillary thyroidectomy in Europe. Curr Surg Rep 2017; 5:17.
2. Kuntscher MV, Erdmann D, Boltze WH, et al. Use of a free jejunal graft for oesophageal reconstruction following perforation after cervical spine surgery: case report and review of the literature. Spinal Cord 2003;41:543-8.

3. Seidenberg B, Rosenak SS, Hurwitt ES, et al. Immediate reconstruction of the cervical esophagus by a revascularized isolated jejunal segment. Ann Surg 1959;149:162-71.

4. Doki Y, Okada K, Miyata H, et al. Long-term and shortterm evaluation of esophageal reconstruction using the colon or the jejunum in esophageal cancer patients after gastrectomy. Dis Esophagus 2008;21:132-8.

5. Razdan SN, Albornoz CR, Matros E, et al. Free jejunal flap for pharyngoesophageal reconstruction in head and neck cancer patients: an evaluation of donor-site complications. J Reconstr Microsurg 2015;31:643-6.

6. Kelly MF, Spiegel J, Rizzo KA, et al. Delayed pharyngoesophageal perforation: a complication of anterior spine surgery. Ann Otol Rhinol Laryngol 1991;100:201-5.

7. Ballesta-Lopez C, Vallet-Fernandez J, Catarci M, et al. Iatrogenic perforations of the esophagus. Int Surg 1993;78:28-31.

8. Attar S, Hankins JR, Suter CM, et al. Esophageal perforation: a therapeutic challenge. Ann Thorac Surg 1990;50:45-9.

9. Pollock RA, Purvis JM, Apple DF Jr, et al. Esophageal and hypopharyngeal injuries in patients with cervical spine trauma. Ann Otol Rhinol Laryngol 1981;90:323-7.

10. English GM, Hsu SF, Edgar R, et al. Oesophageal trauma in patients with spinal cord injury. Paraplegia 1992;30:903-12.

11. O’Brien CJ, Lee KK, Stern HS, et al. Evaluation of 250 freeflap reconstructions after resection of tumours of the head and neck. Aust N Z J Surg 1998;68:698-701.

12. Cho BC, Kim M, Lee JH, et al. Pharyngoesophageal reconstruction with a tubed free radial forearm flap. J Reconstr Microsurg 1998;14:535-40.

13. Laing TA, Van Dam H, Rakshit K, et al. Free jejunum reconstruction of upper esophageal defects. Microsurgery 2013; 33:3-8.

14. Bhathena HM. Free jejunal transfer for pharyngo-esophageal reconstruction. Acta Chir Plast 2002;44:120-3.

15. Coleman JJ 3rd, Searles JM Jr, Hester TR, et al. Ten years experience with the free jejunal autograft. Am J Surg 1987; 154:394-8. 